\title{
A Novel Framework for Supervised Mobile Assessment and Risk Triage of Skin Lesions
}

\author{
Luís Rosado, Maria João M. Vasconcelos, Fernando Correia, Nuno Costa \\ Fraunhofer Portugal AICOS \\ Porto, Portugal \\ luis.rosado@fraunhofer.pt
}

\begin{abstract}
Mobile Teledermatology is a promising tool with the potential to empower patients to adopt an active role in managing their own health status. The main objective of this work is to create a mobile-based framework for risk triage and early diagnosis of skin cancers, with the active involvement of an online community of dermatologists. The presented prototype is composed by the following components: 1) Mobile Application for the patients; 2) Back-end Server that hosts the image processing module and database; and 3) Web Interface for the dermatologists. Using the mobile application, the patients can send a check-up to the back-end server, composed by an image and additional information of the considered skin lesion. The check-up image is automatically analyzed using supervised classification, according to the ABCD Rule and Overall Risk. The system generates an analysis report for each check-up, which will assist the doctors in the check-up risk assessment. After the doctor's validation, a report is sent to the patient's mobile application, and the validated data is used to retrain the supervised classifiers. The overall system architecture and functionalities are briefly described.
\end{abstract}

Keywords-Malignant Melanoma Detection; $A B C D$ Rule of dermatoscopy; Mobile Monitoring; Image analysis

\section{INTRODUCTION}

The incidence rate of skin cancer in the Caucasian population is alarming according to recent statistics [1]. Although Malignant Melanoma (MM) is responsible for most skin cancer related deaths, and the early diagnosis of MM is extremely important considering the high success rates of recovery if the malignancy is detected during the early stages of its development. Patients should consult regularly their dermatologists for skin examinations, and between these appointments they are advised to check for relevant changes in their skin lesions. Patient-oriented approaches are new paradigms for skin lesion analysis, trying not only to motivate and educate the patients, but also to improve the relationship and communication between patients and dermatologists, that can lead to a significant impact in early diagnosis of MM.

Many efforts have been made to design accurate Computer Aided Diagnosis (CAD) systems capable of automatically analyzing skin lesions [2], mostly designed for dermoscopic images [3] and some using macroscopic (clinical) images of skin lesions [4]. The most common criteria that clinicians and dermatologists follow to determine the risk of a given lesion being $\mathrm{MM}$ is the ABCD rule of dermatoscopy [5]. It addresses quantitatively the issue of whether a lesion is benign, suspicious or highly suspicious of $\mathrm{MM}$ according to the analysis of four visual features of the skin lesion: Asymmetry (A), Border (B), Color (C) and Differential Structures (D), being this last criterion only visible using dermoscopy.

\section{SYSTEM ARCHITECTURE}

The system architecture is formed by 3 main blocks (see Figure 1):

1) Mobile Application: used to acquire a skin lesion and send it to the back-end server. The communication between the smartphone and the server was developed in Android 4.0, using the HTTP communication protocol.

2) Back-end Server: consists on a RESTFul webservice implemented in Java using the Jersey library [6] and deployed on an Apache Tomcat 7.0 Web Server running on Ubuntu 10.04. The Database management system was implemented in MySQL using the JDBC DriverManager to handle with the communication between Server and Database. The Image Processing Module (IPM) was implemented in C++ using the OpenCv library [7], which is executed as an external program when an image is received in the server.

3) Web interface: used by the dermatologists to validate the submitted check-ups. Developed with the web application framework Grails 2.2.4 using the Groovy programming language, and deployed on an Apache Tomcat 7.0 Web Server running on Ubuntu 10.04

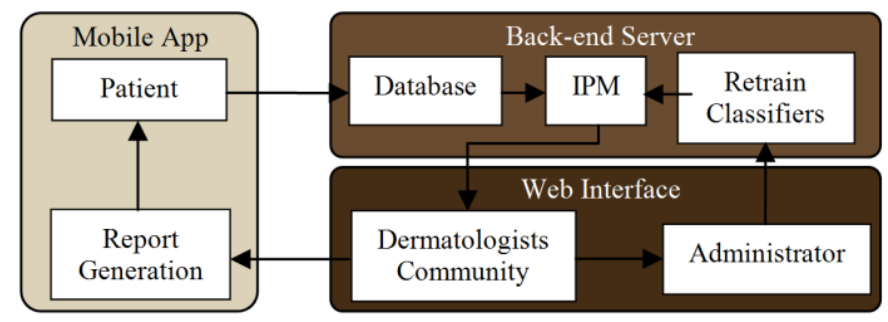

Fig. 1. System architecture workflow. Using the mobile application, the patients can acquire and send a check-up of a skin lesion to the back-end server, where it is saved on the database and automatically analyzed. The system generates an analysis report for each check-up, which will assist the doctors in the risk assessment of that skin lesion. After the doctor validates the analysis, a report is sent to the patient's mobile application. The validated data is used to retrain the machine-learning classifiers used by the IPM. 


\section{FRAMEWORK FUNCTIONALITIES}

In this section, the framework functionalities are briefly described.

The Mobile Application was built for the patients, see Figure 2. Here, the patients can capture the check-up images of their skin lesions or even load the images from the mobile gallery. Each patient can add several skin lesions and each skin lesion can have various check-up images along time. For each skin lesion the patient also indicates its localization, size and can add some additional comments, like itching or redness, to transmit to the doctor. Then, after the doctor's validation, the system sends a report to the patient's mobile application. In this way, the patient can keep a record of the lesions and its reports.

The Back-end Server is composed by the database and the IPM. The IPM analyzes different features of the skin lesion using supervised classification [8] to assess the Asymmetry, Border and Color criteria score according the ABCD rule, as well as the Overall Risk of the skin lesion. The IPM classifiers were trained with a balanced dataset of 80 images that was manually annotated by a dermatology specialist [9]. When the patient sends the check-up image to the Back-end Server, the IPM performs the segmentation of the skin lesion [10] and in case of failure, it asks the patient to capture new image.

The Web Interface can be used by doctors and the administrator. Doctors receive the check-up images loaded by the patients together with the automatic analysis provided by IPM, where it is possible to validate or correct the results, see Figure 3. Each doctor can keep a record of the patients and respective skin lesions. The administrator has the possibility to add new doctors to the database as well as observe the statistics that compare the automatic analysis with the doctor's analysis. In this way, the administrator can realize the accuracy obtained by the automatic analysis and also add this new data to IPM and retrain the supervised classifiers.

\section{CONCLUSIONS AND FUTURE WORK}

The main objective of this work is to create a mobile-based framework for risk triage and early diagnosis of skin cancers, with the active involvement of an online community of dermatologists. The main differentiating factor of this framework is the usage of mobile devices for the capture of skin lesion images, while the specialist's community feedback is used to retrain the supervised classifiers. The proposed framework is an adaptable tool that not only continuously learns with the dermatologist community, but also empowers patients to adopt an active role in managing their own health status.

At the moment, efforts are being made to enlarge the dataset in order to obtain more accurate results and to be possible to test the application in a real case scenario.

\section{ACKNOWLEDGMENT}

This work was done under the scope of the project "SMARTSKINS: A Novel Framework for Supervised Mobile
Assessment and Risk Triage of Skin Lesions via Non-invasive Screening”,PTDC/BBB-BMD/3088/2012 financially supported by Fundação para a Ciência e a Tecnologia in Portugal.

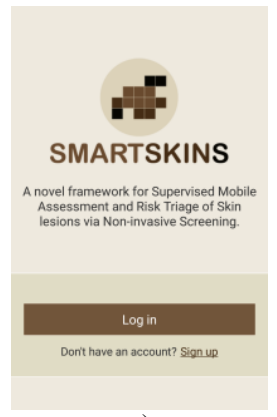

a)

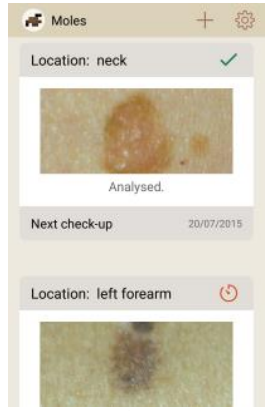

b)

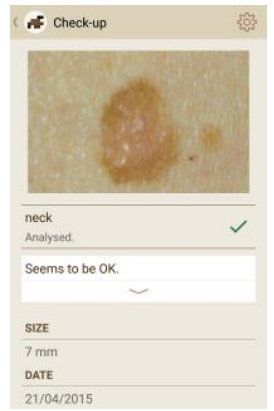

c)
Fig. 2. Mobile application screenshots: a) main menu; b) lesions list; and c) skin lesion analysis report.

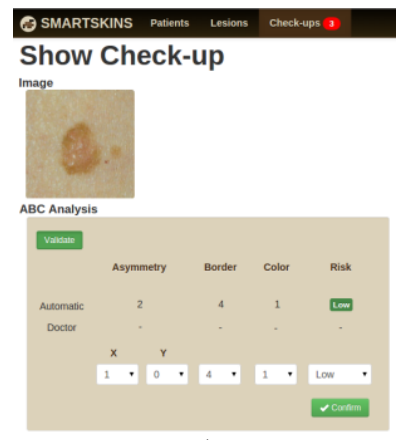

a)
Retrain Classifiers

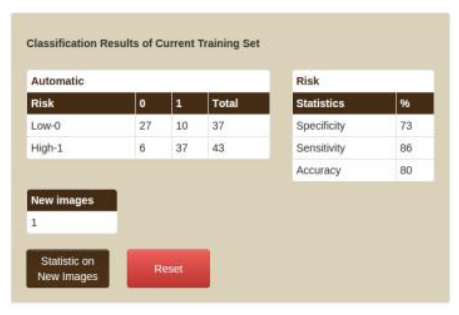

b)

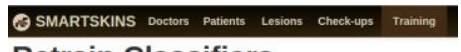

Fig. 3. Web interface screenshot: a) doctors view and b) administrator view.

\section{REFERENCES}

[1] Who [Online 2015], "World Health Organization", http://www.who.int/.

[2] M.E. Celebi,W.V. Stoecker, and R.H. Moss, "Advances in skin cancer image analysis," Computerized Medical Imaging and Graphics, vol. 35, no. 2, pp. 83-84, 2011.

[3] A. Masood, A. Ali Al-Jumaily, "Computer aided diagnostic support system for skin cancer: A review of techniques and algorithms," Int. journal of biomedical imaging, article ID 323268, 22 pages, 2013.

[4] K. Korotkov and R. Garcia, "Computarized Analysis of pigmented skin lesions: a review", Artificial intelligence in medicine 56(2), 69-90, 2012.

[5] W. Stolz, A. Riemann, A. Cognetta, L. Pillet, W. Abmayr, D. Holzel, et al., "Abcd rule of dermatoscopy-a new practical method for early recognition of malignant-melanoma," European Journal of Dermatology, vol. 4, pp. 521-527, 1994.

[6] Jersey, the open source [online] JAX-RS RESTful Web services. http://jersey.java.net/. [Last accessed: March 2015].

[7] G. Bradski, A. Kaehler, "Learning OpenCV: Computer Vision with the OpenCV Library," O'Reilly Media Inc., 2008.

[8] L. Rosado, M.J.M. Vasconcelos and M. Ferreira, "Pigmented Skin Lesion Computerized Analysis via Mobile Devices", $31^{\text {st }}$ Spring Conference on Computer Graphics, Slovakia, 2015.

[9] P.A. Fraunhofer, [online] Smartskins, http://smartskins.projects. fraunhofer.pt/. [Last accessed: April 2015].

[10] L. Rosado and M.J.M. Vasconcelos, "Automatic segmentation methodology for dermatological images acquired via mobile devices", Proceedings of $8^{\text {th }}$ International Conference on Health Informatics, Lisbon, 2015, pp.246-250. 